\title{
Classification and characteristic of maturity groups of Chinese landraces of soybean [Glycine max (L.) Merr.]
}

\author{
Wang Yuesheng · Gai Junyi $\cdot$ Zhang Mengchen · \\ Wang Jian · Chang Ruzhen
}

Published online: 15 June 2007

(C) Springer Science+Business Media B.V. 2007

\section{Erratum to: Genet Resour Crop Evol (2004) DOI: $10.1007 / \mathrm{s} 10722-004-5731-y$}

Please note that the names, order and affiliation of the authors should be:

Wang Yuesheng ${ }^{1}$, Gai Junyi ${ }^{1}$ *, Zhang Mengchen ${ }^{2}$, Wang Jian ${ }^{3}$, Chang Ruzhen ${ }^{4}$

${ }^{1}$ Soybean Research Institute, Nanjing Agricultural University, Natural Center of Soybean Improvement, Minister of Agriculture, Nanjing 210095, China; ${ }^{2}$ Food and Oil Research Institute, Hebei Agricultural

The online version of the original article can be found under doi: 10.1007/s10722-004-5731-y

W. Yuesheng · G. Junyi ( $₫)$

Soybean Research Institute, Nanjing Agricultural University, Natural Center of Soybean Improvement, Minister of Agriculture, Nanjing 210095, China e-mail: ssri@njau.edu.cn

W. Yuesheng

e-mail: wysh@hust.edu.cn

\section{Z. Mengchen}

Food and Oil Research Institute, Hebei Agricultural Academy of Sciences, Shijiazhuang, Hebei 050031, China

\section{W. Jian}

Soybean Research Institute, Northeast Agricultural University, Harbin 150030, China

\section{Ruzhen}

Institute of Crop Germplasm Resources, Chinese

Academy of Agricultural Sciences, Beijing 100081, China
Academy of Sciences, Shijiazhuang, Hebei 050031, China; ${ }^{3}$ Soybean Research Institute, Northeast Agricultural University, Harbin 150030, China; ${ }^{4}$ Institute of Crop Germplasm Resources, Chinese Academy of Agricultural Sciences, Beijing 100081, China; *Author for correspondence (E-mail: wysh@hust. edu.cn, ssri@njau.edu.cn).

Instead of

Wang Yuesheng ${ }^{1}$, Qin Jianbing ${ }^{1}$, Gai Junyi $^{2}$ and He Guangyuang ${ }^{1, *}$

${ }^{1}$ China-UK HUST-RRes Crop Genetic Engineering and Genomics Joint Laboratory, College of Life Science and Technology, Huazhong University of Science and Technology, Wuhan 430074, China; ${ }^{2}$ Soybean Research Institute, Nanjing Agricultural University, Nanjing 210095, China; *Author for correspondence (e-mail: mahh_hust@163.com) 